\title{
Reflectance or Transmission Spectroscopy
}

National Cancer Institute

\section{Source}

National Cancer Institute. Reflectance or Transmission Spectroscopy. NCI Thesaurus.

Code C19340.

The spectroscopic analysis of light reflected from a polished sample. The peaks of reflectance/transmittance occur in the same spectral region where the sample absorbs light. In near infrared (IR) reflectance/transmission spectroscopy (NIRS), the reflectance signals result from bending and stretching vibrations in molecular bonds between carbon, nitrogen, hydrogen, and oxygen. (from FRT R Field Sampling and Analysis Technologies Reference Guide (online)) 